\title{
Desarrollo del lenguaje y control de las acciones: En torno a la regulación verbal
}

\section{Juan Daniel Ramírez*}

Universidad de Sevilla

\section{INTRODUCCION}

El propósito de este artículo es el de exponer algunas ideas aportadas por las investigaciones occidentales, en torno a la regulación de la actividad, siguiendo una línea de trabajos iniciada en la Unión Soviética por A. R. Luria (1969, 1973, 1974, 1984) e inspirada por L. S. Vygotski (1934/1973). En esta misma dirección, pretendemos cubrir varios objetivos, como son: hacer un amplio desarrollo de los aspectos cognitivos de la actividad voluntaria; determinar la influencia que, sobre dichos aspectos, ejerce el habla reguladora; y, también, presentar otras formas alternativas de regulación y control sugeridas indirectamente por algunos investigadores actuales (Bronckart, 1981; Bronckart y Ventouras-Spycher, 1979; Zivin, 1979; Frauenglass y Díaz, 1985). Igualmente, queremos exponer nuestra visión personal de los aspectos impulsivo y activadores de habla reguladora y abordar una posible explicación de sus orígenes. Una vez analizados estos aspectos en profundidad, presentaremos aquí nuestra concepción al respecto, que denominamos «teoría de la modulación de activación». Dicha teoría trata de profundizar en el plano más adaptativo de los procesos ligados a la función impulsiva y a la «activación» (arousal) del habla reguladora, sin los cuales es imposible entender la importancia que esta habla ejerce desde una perspectiva atencional.

Consideramos especialmente importante cubrir estos aspectos, porque a nuestro juicio la teoría y los trabajos de Luria se han estudiado siempre desde perspectivas sesgadas, sin tomar en consideración que su posición es muy amplia, encabalgada entre la psicología evolutiva y la neuropsicología. Dado el escaso conocimiento que tenemos de la psicología soviérica, nos hemos acercado a ella con apriorismos sacados de nuestras propias tradiciones científicas. Esto ya sucedió a comienzos de los años sesenta, cuando el conductismo mediacionista creyó ver en Luria su tabla de salvación (Wozniak, 1972). Nos atreveríamos a decir que algo similar viene a suceder hoy en día. Es posible que la mayoría de los investigadores que abordan el estudio de la obra de Vygotski o cualquiera de sus seguidores, lo haga en base a posicionamientos previos (psicolingüísticos, cognitivistas, etc.) que son científicamente honestos pero que, llegado el caso, pueden confundir más que ayudar. No hemos de olvidar que el punto de partida de los psicólogos soviéticos es un concepto holista, el de «actividad» (Leon-

* Dirección del autor: Universidad de Sevilla, Facultad de Filosofía y Ciencias de la Educación, Sección de 
tiev, 1981). Sin embargo, para salir de la contradicción entre holismo y reduccionismo es importante definir los distintos niveles funcionales que caracterizan a la actividad y al organismo que la produce (acción y operación). Esto sólo es posible a través de una noción sistémica que permita aunar planos contradictorios en apariencia.

Con mucha frecuencia se olvida la concepción sistémica y, por lo tanto, integradora que caracteriza a la teoría y a los trabajos de Luria. Cuando expone su concepción de «sistema funcional complejo», establece las bases para una mejor comprensión de la noción de actividad. Luria considera que un sistema funcional complejo debe poseer dos características fundamentales: la primera, que el sistema ejecute tareas constantes o invariables a través de mecanismos variables, que conducen al proceso a un resultado igualmente invariable; la segunda, que pueda manejar una serie de impulsos aferentes - de ajuste - y eferentes -de realización- (Luria, 1979; pág. 28). Efectivamente, no sólo es importante el hecho mismo de la realización de la actividad, sino, sobre todo, el que el individuo conozca en una información de retorno, el resultado o el efecto de las acciones, de manera que pueda regularlas y autocorregirlas. Para que un sistema así funcione, es necesario contar con un buen formato en el que se puedan representar las acciones que componen la actividad como un todo, con el que podamos comparar los efectos y si éstos se ajustan al plan establecido de funcionamiento. A este respecto, hemos de decir que los trabajos de Luria demuestran que el lenguaje es un buen instrumento facilitador del desarrollo de tal sistema de control y regulación. Representa un buen formato para describir el plan de acción a seguir (Harris, 1979), al facilitar, entre otras cosas, el recuerdo de los objetivos perseguidos (Meacham, 1978, 1979) $)^{1}$.

Pero además de los aspectos representacionales que aporta el habla reguladora, se deben considerar, muy especialmente, los atencionales, no menos importantes 'para el cumplimiento de la actividad. La atención fue un problema tomado en consideración por Luria y sus cols., si bien su análisis se centró, sobre todo, en los aspectos negativos, es decir, en la ruptura del proceso atencional causada por las respuestas de orientación. Un análisis más detallado en esta dirección, tal vez hubiera resuelto algunos equívocos $\mathrm{e}$ indefiniciones descubiertos posteriormente. Entre los investigadores que han estudiado la falta de coordinación entre las respuestas verbales y motoras (Wozniak, 1972; Bronckart, 1973; Bloor, 1977) han surgido críticas a propósito del método motor combinado desarrollado por Luria (basado en la técnica de Ivanov-Smolensky). En algunos casos, porque se considera que los fallos en la regulación verbal proceden de la limitación del canal de elaboración y ejecución de respuesta (Bloor, 1977); en otros, porque se piensa que la metodología desarrollada es, por sí misma, distractora (Bronckart, 1973). Efectivamente, las bolas de caucho se emplean generalmente en otros contextos y activando otros esquemas sensomotores (lanzar contra la pared, botar, etc.), en los que no es frecuente la simple acción de apretar.

A nuestro juicio, nos encontramos ante un problema atencional, que se debe analizar en su totalidad. Habitualmente, los trabajos sobre la atención se centran en los recursos cognitivos que el niño puede manejar, olvidando, sin embargo, que en la atención se deben contemplar dos factores en juego: de un lado, el procesamiento de la información y la elaboración de respuesta; de otro, los componentes motivacionales y activacionales de la conducta. Cuando analizamos la dimensión atencional de la regulación únicamente desde la vertiente cognitiva, sólo observamos la di- 
ferencia que existe entre el niño y el adulto; mientras que el primero tiene evidentes dificultades para coordinar dos acciones de distinta naturaleza (verbal y motora) aunque complementarias entre sí, el segundo puede efectuar acciones sin ningún tipo de relación entre sí. Podemos pensar en un problema complejo mientras que realizamos una tarea que puede ser igualmente compleja, pero sobre la que hemos logrado un cierto grado de automatismo (p. ej., pensamos en algo que nos preocupa mientras conducimos).

La atención está determinada por factores ligados a la motivación y a la activación (arousal) y éstos, a su vez, nos orientan hacia diversos aspectos entre los que cabe destacar la novedad de los estímulos, que, como vamos a ver, frecuentemente comporta la pérdida total de regulación de la acción. Así, un objeto realmente novedoso para el niño generará respuestas de orientación y, con ellas, una serie de acciones desinhibidas que quedan fuera del control voluntario. De modo que un proceso que tendría que estar dirigido desde arriba (conceptual driven), entra en conflicto con otro que se genera desde los datos aportados por el propio objeto (data driven). Esto no nos debe extrañar, puesto que en el artículo introductorio a este dossier, se ha visto ya con qué frecuencia el niño queda fuera del curso de la tarea. Siguiendo a Moreno (1982) se puede enunciar un principio general sobre el valor incentivo expresado en la novedad del estímulo, según el cual la novedad tiende a romper el «equilibrio estimular interno»; esta ruptura induce al organismo a la elicitación de respuestas de orientación dirigidas a la fuente de novedad y, por lo tanto, tendentes al reconocimiento y exploración del estímulo. De este modo, se logra recuperar el equilibrio perdido (Moreno, 1982; pág. 313). La noción de equilibrio estimular interno explicita a la perfección el elevado número de respuestas de orientación contrarias al curso de la actividad que Luria ha podido observar.

Pero estos aspectos activacionales que operan en la atención, no siempre lo hacen en sentido negativo o disruptor del curso de la acción. Lo que vamos a tratar de explicar más adelante es que el sujeto a lo largo del desarrollo logra subordinarlos al sistema y poner la activación al servicio del plan de acción establecido. Esto nos invita a desarrollar un esquema amplio en el que creemos se respeta el objetivo final pretendido por Luria. Se trata, pues, de no romper la unidad del problema, y para eso es necesario ampliar la teoría más allá de los terrenos exclusivamente cognitivos, incorporando los aspectos que van ligados a la atención, entre los que destacamos, fundamentalmente, la cuestión de la activación.

\section{SISTEMAS ALTERNATIVOS A LA REGULACION VERBAL}

Los trabajos de Luria se han centrado fundamentalmente, en el papel que juega el habla en la regulación de la acción. Sin embargo, los psicólogos occidentales que han investigado este tema, presuponen la existencia de otras formas de regulación (Zivin, 1972; Bronckart y VentourasSpycher, 1979; Fuson, 1979; Frauenglass y Díaz, 1985). Bronckart y Ventouras-Spycher (1979) apuntan la posibilidad de una regulación de carácter sensomotor, basándose en que el niño puede realizar ciertas tareas, a partir de los indicios perceptivos que aportan los estímulos incluidos en ellas. Este planteamiento es coherente con los hallazgos de Zivin (1972), que van en dirección opuesta a la regulación verbal. Zivin pudo comprobar que los niños de 4 años de edad verbalizaban escasamente cuando se 
tenían que enfrentar a tareas con un fuerte componente sensomotor (laberintos de seguimiento digital, por ejemplo); en estos casos, los niños que trabajaban en silencio, operaban con más eficacia que aquellos otros que hablaban durante el desarrollo de la tarea. Además, esta misma investigadora observó que muchas de las verbalizaciones producidas por los niños más habladores, eran irrelevantes para el curso de la acción y algunas de ellas simplemente reflejaban reacciones emocionales ante los errorés cometidos. Algunos de los hallazgos más recientes de Frauenglass y Díaz (1985) apuntan también en esta dirección. Todo ello nos conduce hacia una interesante reflexión: no toda el habla privada es, en sí misma, habla reguladora.

En opinión de Fuson (1979), hay datos suficientes para contradecir la hipótesis soviética, que tiende a ver al habla como el único sistema para regular acciones. Así pues, Fuson considera que el lenguaje es uno de los posibles sistemas de control y regulación entre otros varios. Esto se hace patente cuando se observa que tareas de tipo manipulativo (laberintos, ciertos puzzles, etc.) se pueden resolver sin verbalización alguna, utilizando sólo los recursos perceptivos de la propia tarea.

En esta búsqueda de vías alternativas a la regulación verbal, los trabajos de Mischel y cols. (Mischel y Ebbesen, 1970; Mischel y Moore, 1973; Patterson, 1982) ofrecen un interesante ejemplo de autorregulación a partir de representaciones simbólicas o icónicas. Los trabajos del grupo de Mischel demostraban, también, el papel de los factores motivacionales en los procesos de autorregulación. En uno de ellos, se pretendía obtener un tiempo largo de demora, en la consecución de un premio o reforzador, mediante la presencia de un sustituto icónico del premio. Mischel y Ebbesen (1970) ofrecían dos premios de diferente atractivo a niños de 4 años de edad. El más importante de ellos se componía de tres bombones de chocolate, el segundo, de menor cuantía y calidad, consistía en una simple galleta. Como era de esperar, todos los niños preferían el primero cuando se les daba a elegir entre uno y otro. Para alcanzar el premio más importante el niño tenía que permanecer solo, en una habitación y en presencia de ambos premios, por espacio de quince minutos. Muy pocos niños lograban alcanzar el tiempo establecido, ya que la presencia de las golosinas, en lugar de motivar, era causa de frustración. Por el contrario, si en lugar de la visión directa de los premios, lo que el niño tenía delante era un dibujo o una fotografía de los mismos, entonces lograba dominar la situación y alcanzar el tiempo establecido.

Los trabajos de Mischel y cols., se orientan hacia el estudio de la motivación y la sitưación planteada no es la consecución de una acción, sino un tiempo de espera y de inacción. Sin embargo, no cabe la menor duda que esta investigación deja entrever un tipo de regulación que no es verbal, sino de naturaleza simbólica, en el sentido que Piaget (1973) daba al término ${ }^{2}$.

De todos 'modos, en las investigaciones occidentales se ha podido detectar la intervención del habla reguladora incluso en tareas de marcado carácter sensomotor (Zivin, 1973; Goodman, 1981; Frauenglass y Díaz, 1985). Goodman comprobó que en el montaje de puzzles se observa una mayor eficacia, cuando las tareas iban asociadas a altas tasas de verbalización con fines reguladores. Igualmente, Zivin encontró datos que parecían contradecirse con los hallazgos anteriores ya comentados, al detectar una relación positiva entre el empleo de verbalizaciones y el cambio de dirección en laberintos digitales. Frauenglass y Díaz descubren verbalizaciones de habla privada en tareas perceptivas, para las que en principio no es ne- 
cesaria una regulación verbal. Todo ello nos induce a pensar que, efectivamente, existen otras formas de regulación, aparte de la derivada del lenguaje (habla privada o reguladora). También es posible que estas formas de regulación, verbales y no verbales, se vayan superponiendo a lo largo del desarrollo, en una disposición muy similar a los estadios de la representación de estudios por Piaget.

\section{ONTOGENESIS DEL HABLA IMPULSIVA}

A nuestro juicio, el concepto de impulsividad es quizás el .peor estudiado dentro de las investigaciones soviéticas y occidentales. Todos los trabajos tratan de explicar cómo el niño logra liberarse de los rasgos más impulsivos de su conducta, hasta alcanzar un eficaz grado de racionalidad. Aunque nunca se explicita, en las investigaciones occidentales se trabaja con una noción del individuo que, posiblemente, esté mucho más próxima de lo que parece, a la idea de sujeto epistémico. Preocupados por la racionalidad de la conducta, los investigadores olvidan a veces aspectos tan importantes como son los motivos de la conducta del niño o los niveles de activación que facilitan el desarrollo de las acciones. Con bastante frecuencia, también, en estos trabajos sólo se exploran los aspectos cognitivos del habla y de la tarea, violándose así una de las características centrales de la psicología soviética; esto es, que el objeto de conocimiento de la psicología es la «actividad», un concepto molar, en el que quedan integrados los factores cognitivos, motivacionales y activacionales. Para superar estas limitaciones se debe revisar en profundidad algunos aspectos del habla impulsiva, como por ejemplo: el significado de la impulsividad en la conducta infantil; los factores anteriores que la hicieron posible; y, sobre todo, su evolución posterior. Pero para entender estas cuestiones es necesario volver al comienzo de las polémicas que dieron lugar a los trabajos sobre regulación verbal. Como ya sabemos, Vygotski, al contrario que Piaget, consideró por separado los orígenes del pensamiento y del lenguaje. Para Vygotski el pensamiento tiene un origen «no-verbal» y el lenguaje un origen «no-intelectual» (Vygotski, 1934/1973, pág. 78). La posterior integración de ambos permite que el pensamiento sea comunicable y que el lenguaje adquiera dimensiones intelectivas.

En el habla privada parecen sintetizarse dos funciones: la función «cognitiva», la más estudiada; y la función «expresiva», que incluye las verbalizaciones de tipo motivacional y afectivo. Observar el desarrollo del lenguaje sólo desde uno de estos dos planos es falsear el problema, pues a lo largo de su evolución se van integrando progresivamente la función «cognitiva» y representacional localizada en el individuo, con la función «social», «relacional»y, por lo tanto, «afectiva» que no se localiza en el sujeto concreto sino en la interacción (Bronckart y Ventouras-Spycher, 1979, pág. 114). Como apuntan Bronckart y cols., el pensamiento, que puede comunicarse, es el producto de la interconexión de dos tipos de esquemas: los esquemas «representacionales» y los esquemas «comunicativos». Los primeros son el resultado de la inteligencia sensomotora o pensamiento no verbal y los segundos nacen en el contexto de las relaciones afectivas (Bronckart y Ventouras-Spycher, 1979; pág. 115). Mientras que los primeros han sido muy estudiados en el pasado, los esquemas comunicativos y las relaciones afectivas que los generan son menos conocidos. Podemos decir, en general, que la vida emocional está poco estudiada por los psicólogos. Con frecuencia olvidamos que la comunicación humana tiene en su 
origen unos fuertes componentes emocionales, y que estos configuran los primeros intentos comunicativos del niño. Como apuntaba Wallon (1959, 1980) al respecto, las expresiones del bebé son «gestos que se encuentran, sobre todo, en relación con sus estados de bienestar, de malestar o de necesidad: gestos pertenecientes a los sistemas espontáneos de las reacciones afectivas, a la esfera emocional. Bajo la influencia de ese campo emocional, se establecerán rápidamente conexiones entre las manifestaciones espontáneas y las reacciones útiles suscitadas en el entorno» (Wallon, 1980, pág. 112).

El papel que la emoción juega en el despegue de la vida psíquica, se puede rastrear en la maduración de nuestro cerebro. Las bases fisiológicas de la vida emocional no pueden ser otras que las estructuras subcorticales (Wallon, 1980). Así, pues, la emoción encuentra su soporte material, su «hardware», en las regiones subcorticales, «un conjunto funcional que ha evolucionado en la especie mucho antes que las operaciones de representación, de decisión, que se puede atribuir con carácter más exclusivo a la corteza».(Wallon, 1980; pág. 113).

Esta reflexión aunque sintética es importante, pues a nuestro juicio, el origen de las características más destacadas del estadio impulsivo del habla debe rastrearse en la vida emocional; la prueba de ello está en la falta de control y de inhibición de las reacciones emocionales del niño durante los dos primeros años. Sucede, sin embargo, que con el desarrollo del pensamiento, la emoción, que dio origen a los primeros esquemas comunicativos, se transformó y se subordinó a él y, por lo tanto, a la actividad voluntaria y controlada. No debemos olvidar que, para desarrollar una actividad dirigida a objetivos, es necesaria la subordinación de todos los procesos a su consecución y, también, al mantenimiento de la secuencia de acciones necesarias.

La vida emocional comienza por una forma difusa e incontrolada de reacción al medio (emoción protopática). Más tarde, las reacciones del niño se dirigen a las personas y a las cosas (emoción epicrítica) y, en general, a cualquier estímulo sobresaliente. El deseo de manipular un objeto atrayente prima en el niño sobre cualquier otra cosa, y su duración estará determinada por la habituación al mismo o por la aparición de un nuevo objeto y/o un nuevo deseo.

Si aceptamos que la emoción y las reacciones afectivas que suscita están en el origen de los esquemas comunicativos, no es difícil aceptar igualmente que debe operar en la base del habla impulsiva durante los primeros estadios de la regulación verbal. Todo parece indicar que existe una estrecha relación entre la vida emocional (emoción epicrítica) y el habla impulsiva. Recordemos que el niño no se guía por la instrucción del adulto, sino por el predominio de los rasgos más superficiales de los estímulos (destello de la luz, impresión táctil de la pera de caucho, entonación de la voz y de otros rasgos paralingüísticos del habla adulta, etc.), al igual que sucede en el estadio epicrítico de la vida emocional.

No nos cabe la menor duda, que la impulsividad del habla infantil se conecta con el desarrollo de la emoción de carácter epicrítico y de las relaciones en que ésta se expresa. No hemos de olvidar que las reacciones del niño se deben a las propiedades físicas de la voz humana, y quelde ahí procede su carácter impulsivo y la dificultad para inhibir la reacción iniciada. Con el desarrollo, los rasgos impulsivos de su habla se ponen en parte y sólo en parte, al servicio de sus representaciones y acciones, pues, como se ha podido ver, el habla impulsiva es desencadenante de las acciones, pero carece de la adecuada barrera funcional como para impedir que 
éstas se desorganicen. La inhibición sólo será posible a través de la regulación aportada por el habla semántica.

\section{LA ACTIVACION A TRAVES DEL HABLA: MAS ALLA DEL ESTADIO IMPULSIVO}

Para entender el problema de la regulación a través del habla privada en su totalidad, además de determinar el origen de la impulsividad, hay que analizar también su proyección fuera del estadio del habla impulsiva.

El desarrollo del control y la regulación verbal, trae consigo la superación de la impulsividad. Pero razonemos el problema a partir de un principio biológico fundamental: los procesos y estructuras biológicos casi nunca desaparecen del todo, más bien se transforman y se subordinan a procesos de nivel superior (Koop, 1982; Luque, 1985; Ramírez, 1984). Nuestra hipótesis es que la impulsividad no desaparece de la conducta, sino que se transforma y lo que queda de ella es su versión positiva, la «activación» («arousal»); un proceso necesario para el mantenimiento de cualquier acción dirigida a fines. Mantenemos que es posible controlar el nivel de activación mediante los «feedbacks» articulatorios y sensoriales que produce el habla externa reguladora.

Para comprender el problema en su vertiente «hardware» hemos de considerar. la acción conjunta de dos estructuras neuropsicológicas: la primera cumpliría la función propiamente dicha de control y regulación de la actividad; la segunda, la función de regular el tono y la vigilia. En el primer caso, nos estamos refiriendo a los lóbulos frontales; en el segundo, al sistema reticular activador. Luria fue de los primeros psicólogos que observó clínicamente el papel planificador que cumplen los lóbulos frontales (Luria, 1979, 1980). En opinión de este autor, una lesión en éstos «altera sólo las formas superiores corticales de actuación producidas con la ayuda del lenguaje o, en otras palabras, se alteran sólo las formas superiores de la atención voluntaria» (Luria, 1979; pág. 193). Su maduración en el plano de la ontogénesis viene a producirse en un período que transcurre entre los 4 y los 7 años de edad (Luria, 1979; pág. 185), coincidiendo con la etàpa de desarrollo e interiorización de las destrezas reguladoras del habla privada y mal llamada egocéntrica. Esta correlación temporal entre madurez estructural y desarrollo funcional no es un fenómeno gratuito, pone de manifiesto la imbricación entre función y estructura que muchas veces los psicólogos del desarrollo ignoran.

La investigación clínica ha puesto al descubierto que las lesiones en los lóbulos frontales dejan al sujeto a merced de las respuestas de orientación. En el capítulo introductoriọ de este dossier se puntualizaba reiteradamente que la regulación sólo es posible cuando el sujeto logra vencer las respuestas de orientación que la saliencia o fuerza de los estímulos provoca. El daño cerebral en los lóbulos frontales y, especialmente, en el lóbulo frontal izquierdo, rompe la regulación y deja al individuo que lo sufre a merced de la fuerza de los estímulos, alterando así la normal inhibición de las reacciones de orientación (Luria, 1980; pág. 487). Esto nos lleva a reflexionar sobre el papel activador (arousal) que cumple la formación reticular. La excitación generada en esta zona del cerebro no cumple el principio simple del «todo o nada», sino que se desplaza en gradientes de intensidad variable, de acuerdo con la fuerza o intensidad del estímulo. Su función más conocida es la de mantener el tono óptimo del córtex, graduando así el ciclo de sueño y vigilia. Otra función psicológicamente más 
importante, es la de graduar tanto los umbrales sensoriales, como la amplitud de las respuestas. Esto último indica que el sistema reticular interviene en la determinación del nivel de alerta y en la elicitación de la respuesta de orientación ante la fuerza o novedad del estímulo. Además de estas características, hay que añadir que el sistema reticular activador se encuentra conectado en doble dirección con el córtex frontal; lo que significa que pueda activarlo o alertarlo y que, a su vez, recibe por vía descendente su influencia directa. Los lóbulos frontales se convierten así en una doble estructura de control, pues actúa sobre otras áreas del córtex jerárquicamente inferiores; pero a su vez, influye sobre las zonas subtalámicas encargadas del nivel de activación. No es necesario decir que los pacientes con lesiones cerebrales en los lóbulos frontales tiene las mismas dificultades para controlar sus acciones que los niños pequeños del estadio impulsivo descrito por Luria.

Existen evidentes razones de orden neurofisiológico, para mantener la hipótesis de una cierta forma de habla activadora, que configuraría la expresión positiva del habla impulsiva. Sabemos de las conexiones descendentes que unen los lóbulos frontales con la formación reticular; del papel de organización y planificación de las acciones que, desde la vertiente hard. ware, juega el lóbulo frontal izquierdo; y conocemos, también, la influencia de la formación reticular en la modulación de la activación. La existencia de conexiones descendentes entre ambas partes nos permite suponer que en los lóbulos frontales (especialmente el izquierdo) se generan programas de acción, los cuales influyen sobre la formación reticular encargada de mantener el tono necesarió, en función del tipo de actividad a realizar. En este circuito neurofisiológico es donde encontramos razones para defender el papel activacional del habla, más allá del estadio impulsivo propiamente dicho. Por esa conexión descendente que va desde los lóbulos frontales a la formación reticular, podemos deducir que un sujeto con dificultades para la realización de una determinada tarea, se puede ayudar hablándose a sí mismo. Los «feedbacks» sensoriales y motores le permitirán controlar el nivel activador adecuado en relación a la tarea. El habla dirigida a uno mismo puede ser un medio eficaz para lograr que su atención no se distraiga de la actividad en curso, al suprimir las reacciones de orientación que estímulos ajenos puedan suscitar.

Uno de los aspectos fundamentales para comprender la tesis que defendemos (teoría de la modulación de activación), estriba en la importancia que tiene la coordinación entre el habla impulsiva y las acciones bajo su control. Algunos investigadores han enfatizado este aspecto (Wozniak, 1972; Tinsley y Waters, 1982) al demostrar que gran parte de las réplicas a los trabajos de Luria y cols. se hicieron bajo la óptica conductista-mediacionista imperante en los inicios de los años sesenta (Wilder, 1969; Miller, Shelton y Flavell, 1970). Como se consideraba que la instrucción verbal debía mediar entre el estímulo (p. ej., destello luminoso) y la respuesta (p. ej., tracción sobre la perilla), ésta se intercalaba entre ambos en una secuencia temporal perfecta (estímulo-verbalización-respuesta), que reproducía el siguiente esquema mediacionista: $S-\left(r_{v}-e_{v}\right)-R$. Esta interpretación de la teoría de Luria con su consiguiente proyección metodológica no fue afortunada, pues los sujetos experimentales aumentaban el número de errores impulsivos. La experiencia ha demostrado que la verbalización «antes» de la respuesta y «después» de la instrucción, genera desajustes y errores impulsivos. Tinsley y Waters comprobaron que en el estadio impulsivo, los niños logran una mejor ejecución cuando existe una perfecta sincronía temporal entre las respuestas verbal y motora; por el 
contrario, una verbalización que se anticipa brevemente a la acción no hace otra cosa que dificultar el desarrollo de esta última.

Esta perfecta coordinación temporal entre respuestas verbales y motoras, es lo que puede explicar la importancia que adquiere el «ritmo»en algunos juegos infantiles. En ellos hallamos una cierta forma de actividad continua que necesita una coordinación minuciosa de los movimientos (saltar a la cuerda, por ejemplo) y observamos, con frecuencia, que éstos van acompañados de alguna cancioncilla con un ritmo muy marcado, en la que cada golpe de voz se acompasa con un movimiento (levantar un pie, saltar, etc.).

Los componentes rítmicos son frecuentes en los juegos infantiles, y presentan un descenso progresivo entre 5 y 6 años en situaciones de juego libre (Zivin, 1976). Esto parece indicar que, en principio, el ritmo es una ayuda para organizar las acciones y que su empleo desciende en la medida que se adquiere automatismo sobre ellas. Así pues, el ritmo inherente al habla, introduce a su vez, ritmo y organización en otros actos que se realizan simultáneamente (Bronckart y Ventouras-Spycher, 1979; Harris, 1976; Zivin, 1976). Bronckart y Ventouras-Spycher consideran que la regulación sólo es posible entre dos sistemas con componentes motores, uno de los cuales está más desarrollado que el otro. El ritmo de ciertas producciones verbales funciona como sistema temporizador y seriador de la acción continua. Este parece ser el caso de las canciones populares ligadas al trabajo. Algunas canciones del folklore negro americano, los martinetes en el cante flamenco y ciertas canciones del folklore ruso - «Los remeros del Volga», por ejemplo- reflejan una buena manera de armonizar el movimiento con la rima y son un perfecto ejemplo de facilitador y organizador de la actividad. En ninguno de los ejemplos comentados podemos hablar de impulsividad, ya que la acción está perfectamente organizada. Sin embàrgo, algunos de los rasgos impulsores continúan. Ellos constituyen un proceso más complejo conocido como «activación». Si contemplamos muchos de los juegos rítmicos infantiles y de las actividades laborales hoy en desuso, detectamos una forma específica de «habla activadora», a caballo entre las otras dos formas de habla más conocidas: la impulsiva y la semántica. El habla activadora no es impulsiva puesto que colabora con una actividad organizada, pero tampoco es semántica, ya que su significado no es relevante y no tiene por qué presentar relación alguna con las acciones que organiza.

Si tomamos en consideración los aspectos activadores heredados del estadio impulsivo y subyacentes a las acciones motoras, éstos pueden resolver ciertas contradicciones puestas de manifiesto en algunas investigaciones recientes (Saltz, Campbell y Skotko, 1982). Saltz, Campbell y Skotko comprobaron que la verbalización de órdenes sencillas ( «Haz palmas», «no te toques las orejas», etc.) emiridas en voz alta (casi gritando), desorganizan las acciones de los niños más pequeños ( 4 años de edad). Sin embargo, las verbalizaciones de baja intensidad facilitan el desarrollo de sus acciones y reducen el número de errores impulsivos. Por el contrario, los mayores ( 6 años de edad) se beneficiaban de las verbalizaciones realizadas con una alta intensidad, pues cometían un número de errores impulsivos muy por debajo de los que se producían hablando en voz baja. En opinión de estos investigadores, sus datos contradicen la tesis de Luria, ya que los mayores se beneficiaban de una impulsividad que a esas edades, debería estar totalmente superada.

A nuestro juicio, los hallazgos de Saltz y cols., lo que demuestran realmente es el papel activador que puede generarse desde la propia habla y 
que, bien utilizado, puede ser un factor estructurante más de las propias acciones. Para los pequeños, el grito era un desencadenante de su impulsividad; para los mayores, esa fuerte verbalización representa una elevación de la activación, acorde con las condiciones de la tarea. Después de los comentarios desarrollados a propósito del ritmo a través del habla, no es difícil entender nuestra actitud crítica hacia las conclusiones de Saltz y cols. De nuevo el problema no es de impulsividad, sino de nivel de activación y su adecuación al tipo de tarea.

\section{ACTIVACION Y HABLA SEMANTICA}

Resultan sobradamente conocidas las discrepancias que existen entre Piaget (1976) y Vygotski (1973) en la forma de entender el lenguaje egocéntrico; por ello, no es necesario llevar à cabo una revisión en este sentido. Sólo se da entre ambos una débil coincidencia, la falta de conciencia que impide al niño refrenar el habla inadaptada a la comunicación. Todo lo demás son discrepancias entre ambos investigadores. Estas surgen de las diferencias teóricas y metodológicas que existen entre ellos, pues, mientras que Piaget realiza un análisis estructural de las emisiones infantiles, Vygotski toma un punto de partida más funcional. El planteamiento de este último queda perfectamente reflejado en la siguiente cita:

El lenguaje egocéntrico no permanece durante mucho tiempo como un simple acompañante de la actividad infantil. Aparte de ser un medio expresivo y de reflejar la tensión, se convierte pronto en un instrumento de pensamiento, en sentido estricto, en la búsqueda y planteamiento de la resolución de un problema (Vygotski, 1973; pág. 40).

Dos de los aspectos más observados por Vygotski a propósito del habla egocéntrica fueron: la «simplificación» y la «predicatividad». Ambos se complementan mutuamente, pues, si observamos sólo el primer aspecto, veremos exclusivamente la falta de destreza para comunicarse con los demás, que presentan los niños en torno a los 4 años de edad; pero si observamos que estas producciones sólo expresan la parte predicativa de cada oración, entonces comprobaremos la verdadera función cognitiva del hàbla egocéntrica, sobre la que Vygotski apunta lo siguiente:

En un comienzo, la estructura del lenguaje egocéntrico es igual a la del social, pero en el proceso de transformación hacia el interiorizado, se torna gradualmente menos completa y coherente, al quedar gobernada por una sintaxis casi totalmente predicativa (Vygotski, ob. cit.; pág. 188).

Nótese, pues, que este teórico consideraba la interiorización, como un proceso que se acompaña de otro no menos importante, en el que el habla tiende a comprimirse y a adquirir su verdadera función cognitiva, esto es, la función predicativa ${ }^{3}$.

Para estudiar los dos aspectos del habla reguladora (simplificación y predicatividad), Wertsch (1979) propone una interesante forma de análisis, que asume la tesis de Chafe (1976) sobre el papel de las diferentes partes de la oración en el acto comunicativo. Este último considera que la información conocida por los participantes en la comunicación (given), recibe una baja entonación y en muchos casos es pronominalizada; por el contrario, la información que es nueva para el oyente tiende a recibir un mayor énfasis (new). Este aspecto no es nuevo en la psicología del lenguaje, Vygotski lo había enunciado yạ al considerar que en el lenguaje in- 
terior, lo que es conocido por el sujeto tiende a ser omitido y sólo lo nuevo es predicado. De ahí su sintaxis exclusivamente predicativa (Vygotsky, ob. cit.; pág. 188).

Para desarrollar un modelo de gramática centrado en las funciones que cumplen las diferentes partes del habla, es fundamental volver a recuperar la noción de «conciencia» (Chafe, 1976; Wertsch, 1979). Este concepto no va a ser analizado aquí, pero se debe tener en cuenta, ya que en el proceso de regulación, la función del habla no es otra que soslayar lo «dado» ( $g i$ ven) o «conocido», para poner en el foco de la conciencia lo que es «nuevo» (new) y debe ser cognitivamente explorado o usado en el curso de la acción. En el habla reguladora (externa o interna) no existe el oyente, y toda la situación se retrae al sujeto que habla y/o actúa y al objeto del que se habla y/o sobre el que se actúa. En esa situación lo conocido, lo dado, es el objeto, mientras que lo nuevo es aquello que vamos descubriendo en él, o las posibilidades para la acción que nos ofrece. De ahí que dicho objeto no sea nombrado, pues está ante nuestros ojos y en nuestras manos; las que se nombrarán serán sus propiedades más relevantes en el curso del juego o de la tarea. Según nuestra hipótesis, el nivel activador bajará prácticamente a cero en las partes conocidas de la tarea y se elevará en las partes nuevas, pasando el sujeto a denominar los rasgos o propiedades que interesan del objeto o de las acciones.

Así, pues, el planteamiento de Wertsch es perfectamente coherente con la tesis de Vygotski, y esto puede verse con claridad en la siguiente cita del último:

El niño habla de las cosas que ve, oye o hace en un determinado momento; es así como tiende a dejar de lado el sujeto y todas las palabras relacionadas con él, condensando cada vez más su lenguaje hasta que sólo quedan los predicados. Mientras más diferenciada se vuelve la función del lenguaje egocéntrico, más agudas son sus peculiaridades sintácticas: simplicación y predicatividad (pág. 188).

Si queremos dar forma coherente a las oraciones emitidas en ese discurso entrecortado, hemos de mirar al contexto y a la situación total, pues en ellos se encuentran los aspectos ausentes del habla (los sujetos que en su momento se elicitaron). Sólo por la exploración del contexto se puede conocer cuáles son los elementos que se están introduciendo en el campo de la conciencia.

En este momento de la etapa egocéntrica del habla, lo que se detecta en el niño es la exteriorización del plano psicológico, inadaptado a las estrategias de la comunicación, pues no se trata tanto de que se le entienda, como de seguir y controlar el curso de su acción en desarrollo.

\section{HIPOTESIS EN TORNO AL CONTROL SEMANTICO}

A comienzo de los años sesenta, algunos psicólogos de orientación mediacionista (Kendler y Kendler, 1961) que trabajaban en tareas de discriminación y formación de conceptos, observaron que los niños mayores de siete años se guiaban por las propiedades de los estímulos y sus etiquetados verbales. Dicha forma de operar se contraponía a la de los niños de cuatro años, los cuales procedían mediante aproximaciones sucesivas y, por lo tanto, sin ningún tipo de mediación. Estos y otros hallazgos similares desembocaron en la formulación de la hipótesis de «deficiencia de mediación» (Reese, 1962), según la cual el lenguaje tiene consecuencias media- 
tizadoras sobre la conducta del sujeto. Las dificultades que los niños pequeños presentaban en ciertos problemas conceptuales, se debían a la falta de mediación de su lenguaje, que domina en el plano de la comunicación, pero que está ausente del plano cognitivo.

Flavell y sus colaboradores (Flavell, Beach y Chinsky, 1966) desarrollaron una hipótesis alternativa, a partir de una cierta ambigüedad descrita por Maccoby (1964) sobre la hipótesis de Reese. Para Maccoby, la raíz del problema éstá en saber si el niño hace uso de marcas verbales que, por alguna razón, no ejerce ningún efecto mediador o si, por el contrario, no sabe producirlas en el momento adecuado. A partir de esta segunda alternativa, Flavell propuso la hipótesis de «deficiencia de producción», según la cual los niños pequeños no producen espontáneamente las marcas verbales que les permiten guiar su conducta cognitiva.

La hipótesis se veía avalada al demostrar Flavell y cols. en una prueba de memoria, que aquellos niños que nombraban espontáneamente los estímulos, podían recordar mejor que los que no lo hacían.

Pero el debate entre ambas hipótesis no estaba resuelto. En un trabajo posterior (Keeny, Cannizo y Flavell, 1967) se observó que al enseñar a repasar a niños entre 6 y 7 años de edad, éstos podían alcanzar tan buenos resultados como los que repasaban espontáneamente. Sin embargo, algo más de la mitad fallaron en un nuevo ensayo en el que el experimentador no les inducía explícitamente al repaso. Esto venía a demostrar, que enseñar el uso de una destreza no implica, necesariamente, su generalización a otros contextos.

Existen indicios claros que nos permiten pensar en el efecto facilitador de la verbalización, también en el plano de la memoria. No obstante, los hallazgos experimentales aportan pruebas a favor y en contra del uso del habla externa y audible (Bronckart, 1973; Meichenbaum y Goodman, 1979; McCabe, 1979). Meichenbaum y Goodman comprobaron que el habla en voz alta mejoraba la realización de tareas motoras en niños de parvulario, en tanto que dificultaba su curso a las edades de 6 y 7 años; Bronckart (1973) llegó, igualmente, a conclusiones muy similares. Pero quizás, el trabajo más significativo a este respecto, sea el de Meacham (1978, 1979). Este enseñó a niños de edades comprendidas entre 3 y 5 años, a modificar la trayectoria de un triciclo a través de órdenes sencillas que los niños se daban a sí mismos («up» -adelante-, «back» - atrás-y «stop» -alto-). Los resultados demostraron que los niños mayores se implicaban más que los pequeños en el uso de las órdenes verbalizadas por ellos mismos; además, eran infrecuentes las verbalizaciones espontáneas en el grupo de control, cuyos sujetos no habían sido adiestrados en el uso de estas autoinstrucciones. Esto es coherente con el origen externo del control y la regulación verbal y hace de este tema una cuestión de aprendizaje. pero quizás la observación más importante realizada por Meacham, sea que el cambio de una acción inadecuada a otra adecuada a la tarea, era más probable si intervenían las autoinstrucciones. Este último aspecto nos permite, una vez más, interpretar la actividad verbal en términos de esquema TOTE (Miller, Galanter y Pribram, 1960/1983), ya que el niño, a través de las verbalizaciones, puede evocar el objeto de sus acciones y modificar el curso de las mismas. 


\section{SISTEMATIZACION DE LOS FACTORES IMPLICADOS EN EL PROOCESO DE REGULACION DE LA ACCION: TEORIA DE LA MODULACION DE ACTIVACION}

Una vez analizados los aspectos más significativos de los trabajos centrados en regulación de la acción y en campos próximos, es importante proceder a integrar y sistematizar las variables que intervienen en este proceso. Posteriormente, se volverá a algunos de los trabajos que ya se han reseñado, con la finalidad de buscar la razón de las posibles contradicciones surgidas tanto en el ámbito de las investigaciones soviéticas, como en èl de las occidentales.

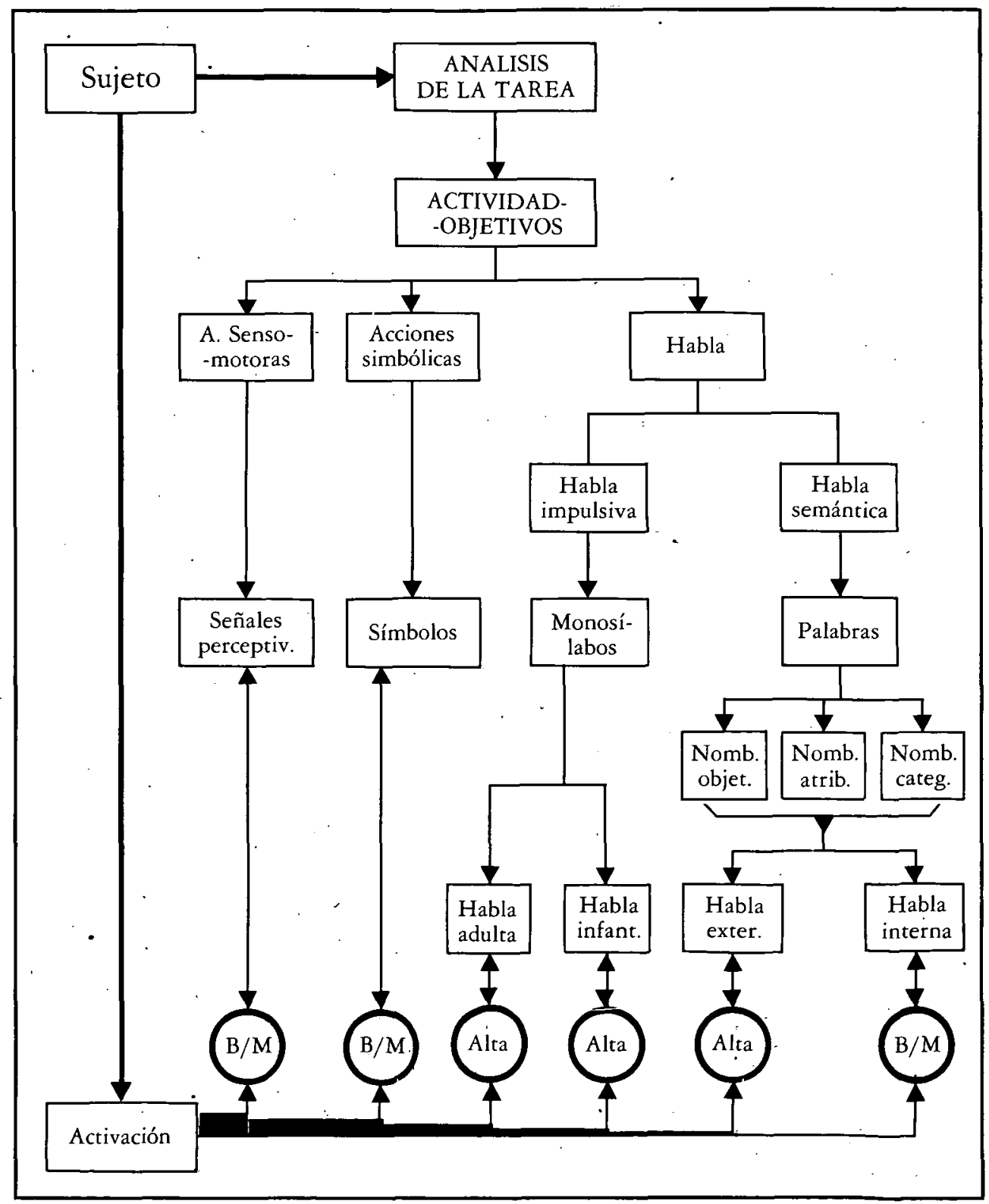


En nuestra teoría se pretende integrar simultáneamente los aspectos «cognitivos» y «activadores» que hacen posible la regulación de la actividad dirigida a fines.

Los procesos implicados en la regulación de las acciones del șujeto se inician con el análisis de la tarea, para continuar después con el establecimiento del tipo de actividad y los objetivos que se pretenden alcanzar. El siguiente paso consiste en explicitar las acciones que configuran el curso de la actividad; y, por último, finaliza con las guías representacionales que facilitan el curso de las acciones. Estas acciones son de tres tipos diferentes: acciones sensomotoras (puzzles y tareas similares), que necesitan apoyanse en señales perceptivas para su realización; acciones guiadas simbólicamente (mediante guías que son símbolos o iconos); y acciones que se explicitan y guían a través del habla. En el habla impulsiva las guías verbales pueden ser simples monosílabos o palabras en las que el significado carece de valor o pasa a un plano secundario.

La activación es un proceso continuo que oscila entre niveles altos y bajos, al contrario que los procesos cognitivos. Una señal perceptiva, por ejemplo, se produce cuando el estímulo supera un determinado umbral; igualmente, una instrucción verbal se puede dar o no dar; en un caso y en otro no caben términos medios. Por el contrario, la activación no va de todo a nada, sino en gradientes de diferente intensidad. No hemos de olvidar que la principal estructura neurofisiológica implicada en el proceso de activación es la formación reticular, una de cuyas funciones es la de elevar o descender los umbrales sensoriales y amplificar los reflejos autónomos.

Para la realización de cualquier tarea, el sujeto necesita primero analizarla y organizar después, adecuadamente, el conjunto de acciones dentro de una actividad que permita alcanzar el fin previsto. Simultáneamente, es necesario organizar el nivel de activación precisa. Una tarea compleja que necesita realizarse en varias fases, precisará de un nivel activador más bien bajo; sin embargo, otra simple y con acciones que se repiten reiteradamente, necesita un nivel alto que impida perder la concentración o el ritmo y coordinación de los movimientos implicados, etc. Se necesita, pues, un justo nivel de activación que permita el desarrollo de la tarea, sin generar situaciones de frustración o ansiedad, contrarias a ella.

Los niveles bajo, medio y alto expresados en los círculos, representan un acercamiento al problema de la activación. Aceptamos su simplicidad, pues, por ahora, sólo significan una aproximación, que pretende introducir una variable ausente de los trabajos de regulación y control de las acciones. Nos referimos, concretamente, al «nivel» o «intensidad» de activación, con frecuencia olvidado en este tipo de trabajos.

En cuanto a las tareas sensomotoras y a las guiadas simbólicamente, se ha calculado su nivel de activación en función del tipo de tarea contemplado aquí (puzzles y tiempo de espera a la gratificación). No dudamos que en un futuro podamos encontrar tareas de estos tipos que puedan exigir un nivel activador alto. En ambos casos, la variable «tiempo» es muy importante, pues no se puede mantener al sujeto excesivamente activado, sin generarle una cierta dosis de frustración.

Como se puede observar en nuestro esquema, los niveles altos se concentran en el habla externa en sus dos formas: habla impulsiva y habla externa semántica. Cuando las tareas tienden a ser monótonas, el sujeto necesita articular monosílabos o palabras que le permitan elevar el nivel de activación. Buenos ejemplos son las voces de mando en la instrucción militar o, como se ha apuntado con anterioridad, las canciones populares 
ligadas al trabajo. Estas verbalizaciones rítmicamente organizadas permiten que el sujeto no pierda el curso de la actividad continua.

En cuanto al habla semántica, Luria propone la existencia de dos estadios: un primer estadio de habla semántica externa, y un segundo de habla interna. Algunos psicólogos occidentales dudan de la existencia del primero. En un cierto sentido coincidimos con esta segunda alternativa y consideramos más bien que la verbalización en voz alta de la orden es necesaria en el plano semántico, cuando la tarea es difícil por sí misma o el sujeto demuestra poca pericia en su realización. En estas condiciones, el sujeto debe elevar el nivel activador para no perder el control. Es entonces, cuando el niño necesita decirse en voz alta la acción a realizar, su objetivo, etc.

A primera vista puede parecer un contrasentido que el sujeto hable en voz alta, tanto en las tareas sencillas como en las complejas, pero las razones son distintas en un caso y en otro. En el primer caso es necesario elevar el nivel activador para vencer las respuestas de orientación contrarias a las órdenes y contrarrestar, así, la monotonía; en el segundo, el sujeto poco diestro necesita hablar para evocar los objetivos y las acciones que los hacen posible, al concentrar la atención en los momentos cruciales de la tarea.

El habla semántica puede actuar sobre las acciones, como hemos visto, pero también lo puede hacer sobre las representaciones del sujeto, especialmente en todo lo concerniente al proceso de codificación en la memoria. Esta dimensión del habla externa quizás no haya sido tan intensamente estudiado; sin embargo, es conocida la eficacia del repaso en voz alta como estrategia facilitadora del recuerdo, puesta de manifiesto por Flavell y sus colaboradores. Este aspecto se recoge en el apartado del habla semántica relativo a «nombrar objetos». Sin embargo, en muchos casos no es tan efectivo nombrar o repasar el estímulo varias veces y sí lo es, desde el punto de vista de la encodificación, nombrar sus atributos o propiedades más significativas. En otras ocasiones puede ser más importante nombrar la categoría de pertenencia («nombrar categorías»), ya que ello permite generar agrupamientos (clusters) que facilitan la recuperación, como lo demuestran los trabajos en el marco de la teoría de los niveles de procesamiento (Craik y Lockhart, 1972; Craik y Tulving, 1985).

«Nombrar atributos» o «nombrar categorías», es decir algo nuevo del estímulo, lo que todavía no ha sido explicitado. Por ejemplo, si se nos presenta una palabra escrita y se nos invita a que digamos su clase de pertenencia o sus propiedades específicas, se nos induce a poner bajo el foco de nuestra conciencia los rasgos distintivos que facilitan su encodificación. Esta novedad que podemos extraer del estímulo y que podemos predicar (Wertsch, 1979), presenta muchos contactos con las tareas de orientación empleadas por Craik y Tulving. Cuando se le pide al sujeto en una prueba de memoria, que diga la clase a la que pertenece un determinado estímulo («rosa», por ejemplo), en cierto modo estamos simulando cómo funciona el habla interna, ya que sólo pedimos que se emita el predicado. No es necesario incluir el sujeto de la oraciọ́n («rosa es una flor») porque ya está presente, es el propio estímulo; lo verdaderamente importante es poner en la conciencia lo novedoso, lo que no viene dado directamente y que en el caso que nos ocupa, permite agruparla con otras palabras de la lista.

Cuando se induce en forma experimental este mismo proceso, en un niño que no repasa espontáneamente, sucede algo más: no sólo se focaliza el elemento significativo (la clase, p. ej.), sino que esta focalización se ve 
facilitada por el nivel óptimo de activación, inducido por el «feedback»articulatorio y sensorial que introduce el habla externa.

Después de lo qué ya hemos apuntado, es posible explicar los fracasos en el empleo del habla semántica externa en diversas investigaciones occidentales. Aparte de que las verbalizaciones extensas inducidas pueden entrar en competición con las acciones motoras en el restringido canal de respuesta de los niños pequeños, puede suceder, también, que la verbalización produzca un nivel de activación demasiado elevado. Abundando en esta misma dirección, hay que señalar otro aspecto fundamental: la predicatividad de que hace gala el habla reguladora. En más de un caso, se puede estar pidiendo al niño que diga muchas cosas sobre la tarea, cuando la verdadera habla reguladora sólo incide en los aspectos novedosos y distintivos que permiten encodificar un estímulo o cambiar el curso de una acción.

\section{CONCLUSIONES}

El objetivo de este trabajo ha sido el de definir e integrar todos los factores implicados en la regulación de las acciones. Las conclusiones que podemos apuntar son las siguientes:

- La idea de regulación implica a la vez las de control y modulación. El control requiere dos cosas: la iniciación de la acción y su inhibición, una vez que el objetivo ha sido alcanzado; la modulación se expresa a través del nivel de activación más adecuada al curso de la acción, para que ésta se lleve a cabo sin sobrecargas que la perturben.

- A tenor de lo apuntado, sólo se puede entender la regulación a través de un sujeto con capacidad para acoplar los planos cognitivo y activador.

- De todas las formas posibles de regulación, la que llega a adquirir más importancia en el curso de la actividad voluntaria, es la regulación a través del habla.

- Los factores impulsivos proceden de los esquemas comunicativos, base del desarrollo del lenguaje. Ambos tienen un origen no-intelectual y emocional. Con el paso del tiempo, se nutren de un sistema de signos adquiridos socialmente.

- De su origen emocional, al lenguaje le queda la dimensión activadora o impulsora de la acción; por su naturaleza social, el lenguaje se constituye en barrera funcional capaz de inhibir la actividad, bien porque se ha alcanzado el objetivo, bien porque su curso no es el adecuado.

- De todo lo apuntado se desprende que el habla es una forma de acción que puede organizar y controlar a otras acciones y, también, que puede modificar las representaciones del sujeto. De ahí su importancia en el desarrollo de la actividad voluntaria.

- De todo lo apuntado se desprende que el habla es una forma de acción que puede organizar y controlar a otras acciones y, también, que puede modificar las representaciones del sujeto. De ahí su importancia en el desarrollo de la actividad voluntaria. 
1 En el artículo introductorio de este dossier se ha enfatizado el papel inhibidor del habla reguladora. Como allí se apuntaba, el lenguaje es un buen formato porque facilita la representación de la acción. Cualquier respuesta que el sujeto emita se confronta, en una información de retorno, con la totalidad de la representación. Por esta razón, es importante la función inhibidora; resultante de la «barrera funcional» que puede llegar a ser el lenguaje. Dicha barrera funcional presenta una curiosa similitud con el factor I de Pascual-Leone. Según éste, «un esquema ejecutivo dominante (el plan de acción o programa dominante en el sujeto) puede inhibir los esquemas en el campo de la activación que son irrelevantes para la implementación del plan en curso» (J. Pascual-Leone: «La teoría de los operadores constructivos, 1978, pág. 211. J. Delval [comp.]: Lecturas en psicología del niño. Vol I. Madrid: Alianza).

2 Según Piaget, «el símbolo se basa en el simple parecido entre el objeto presente que juega el papel de significante y el objeto ausente significado, lo cual implica una representación...» (J. Piaget: La formación del símbolo en el niño. México: Fondo de Cultura Económica, 1973, pág. 137). La relación que se produce entre el significante y su significado es analógica, por lo tanto. En el caso que nos ocupa, esa analogía es total, porque lo que Mischel y sus cols. presentan es una fotografía de los reforzadores. Sin embargo, es lógico pensar que el tiempo de espera se podría obtener igualmente con una representación simbólica menos puntual, mucho más simple en detalles (un dibujo, por ejemplo).

3 En algunas ocasiones, habla externa (comunicativa) y habla interna, privada y/o reguladora coinciden. Una de las mejores observaciones de Vygotsky, pocas veces tomada en cuenta por los estudiosos del habla privada, considera esta posibilidad. Al comentar un texto de Tolstoi, Vygotsky hace la siguiente consideración: «... cuando los pensamientos de los interlocutores son los mismos, el papel del lenguaje se reduce a un mínimo. Tolstoi señala (...) que entre gente que vive en estrecho contacto psicológico, la comunicación a través de formas abreviadas del lenguaje es más bien una regla que excepción» (L. S. Vygotsky: Pensamiento y lenguaje. Buenos Aires: La Pléyade, 1973, pág. 183).

\section{Resumen}

En este trabajo nos proponemos revisar la teoria de Luria en torno a la regulación verbal y otros campos afines de la relación lenguaje-pensamiento, según la perspectiva soviética iniciada por Vygotsky. En esta revisión se pretende efectuar un análisis detallado de las variables que están presentes en el proceso de regulación, y explicar cómo éstas se relacionan entre sí. Para alcanzar tal objetivo se incide en los siguientes aspectos: la doble vertiente cognitiva y motivacional del babla reguladora; las funciones que cumplen los componentes del babla egocéntrica y/o reguladora (Vygotsky, Wertsch); $y$, por último, el origen de los factores activadores del habla, procedentes del estadio impulsivo y de los esquemas afectivos y de comunicación. Esta revisión se completa con la sistematización del proceso de regulación en un conjunto, que incluye las variables implicadas y un análisis de las mismas. A partir de ahi se pasa a comprobar los acuerdos y desacuerdos existentes entre las investigaciones soviéticas y occidentales; $y$ a tratar de dar explicación de los resultados contradictorios en base a nuestra sistematización del proceso regulador, por el que, pensamos, es posible predecir la conducta a seguir por el niño en la realización de distintos tipos de tarea.

\section{Summary}

During the course of this project, we intend to revise the Luria theory with regards to speech regulation and other fields closely associated with language-thought relation, in accordance with the soviet perspective iniciated by Vygotsky. In this revision, it is intended to carry out a detailed analysis of the variables with are pre. sent in the regulation process, and to explain the manner in which these are related one to the otber. With this object in mind, the following aspects are examined: the recognised and motivational double facet of the verbal regulation; the functions of the components of the egocentric and/or regulation speech (Vygotsky, Wertsch); and, to finish, the origin of the verbal arousing factors, which procced from the impulsive state and from certain emotional schemes and from communication. This revision is completed by means of the systemization of the regulation process in its entirety, which inclides the aformentioned variables and an analysis of the same. Aport from this, the existing agreements and disagreements between soviet and western investigations are examined and we try to give an explanation for the contradictory results based on our systemization of the regulating process, by means of which we think it is possible to foretell a child's conduct in the realiza. tion of different types of tasks.

\section{Résumé}

Cet article offre une révision de la théorie de Luria en ce qui concerne la régulation verbale et autres tbèmes en rapport avec la relation langage-pensée, suivant la perspective soviétique initiée par Vygótsky. Cette révision prétend effectuer une analyse détaillée des facteurs présents dans le processus de régulation et expliquer quels sont leurs rapports. Dans ce but on étudie les aspects suivants: les deux versants cognitif et motivationnel de la parole régulatrice; les fonctions que remplissent les composants de la parole égocentrique et/ou régulatrice (Vygotsky, Wertsch); et, pour finir, l'origine des facteurs activeurs de la parole provenant du stade impulsif et des schèmes affectifs et de la communication. Cette révision est complétée par la systématisation du processus 
de régulation dans son ensemble que comprend une analyse des facteurs impliqués. Il s'ensuit la vérification des accords et désaccords existant entre les recherches soviétiques et occidentales. On tente d'expliquer les résultats contradictoires a partir de cette systématisation du processus régulateur qui, on pense, permet de prédire la conduite que l'enfant doit suivre dans la réalisation de différents types de taches.

\section{Referencias}

BLOOR, D.: «The regulatory function of language», en P. J. Morton y J. C. Marshall (eds.): Psycholinguistics, Developmental and patological. Ithaca, Nueva York: Cornell University Press, 1977.

BRonCKarT, J.-P.: «The regulating role of speech: A cognitive approach». Human Development, 1973, 16, 417-439. (Trad.: «Papel regulador del lenguaje». Monografia de Infancia y Aprendizaje: La adquisición del lenguaje. Madrid, 1981, 115-131.)

Bronckart, J.-P., y Ventouras-Spycher, M.: «The Piagetian Concept of Representation and the Soviet-Inspired View of Self-Regulation», en G. Zivin (ed.): The Development of Self-Regulation through Private Speech. Nueva York: Wiley, 1979, 99-131.

CHAFE, W. L.: «Givennes, Contrastativeness, definiteness, subjects, topics, and point of View», en C. N. Li (ed.): Subject and Topic. Nueva York: Academic, 1976

CRAIK, F. I. M., y LOCKHART, R. S.: «Niveles de procesamiento: un marco para la investigación sobre la memoria». Estudios de Psicología, 1981, 2, 93-109.

CRAIK, F.I. M., y Tulving, E.: «Profundidad de procesamiento y retención de palabras en la memoria episódica». Estudios de psicología, 1981, 2, 110-146.

Flavell, J. H; BEACH, D. R., y CHINSKY, J. M.: «Spontaneous Verbal Rehersal in a Memory Task as a Function of Age». Child Development, 1966, 37, 283-299.

FraUENGLASS, M. H., y Díaz, R.: «Self-regulatory functions of children's private speech: A critical analysis of recent challenges to Vygotski theory». Developmental Psychology, 1985, 21, 357-364.

Fuson, K. C.: "The Development of Self-Regulation Aspects of Speech: A Review», en G. Zivin (ed.): The Development of Self-Regulation through Private Speech. Nueva York: Wiley, 1979.

Goodman, S. H.: «The Integration of Verbal and Motor Behavior in Preschool Children». Cbild Development, $1981,52,280-289$.

HARRIS, A.: «The Function of Speech Rithms in the Regulation of Non-Speech Activity», en K. E. Riegel y J. A. Meacham (eds.): The Developing Individual in a Changing World. Vol. I: «Historical and Cultural Issues». La Haya: Mouton, 1976, 172-180.

- «Historical Development of the Soviet Theory of Self-Regulation», en G. Zivin (ed.): The Development of Self-Regulation through Private Speech. Nueva York: Wiley, 1979.

Keeny, T. J.; Cannizo, S. R., y Flavell, J. M.: «Spontaneous and Induced Verbal Rehersal in a Recall Task». Child Development, 1967, 38, 953-966.

KeNDler, H. H., y KeNDIER, T. S.: «Effect of Verbalization on discrimination Reversal Shifts in Children». Science, 1961, 134, 169-1620.

Kopp, C. B.: «Antecedents of Self-Regulation: A Developmental perspective». Developmental Psychology, 1982, $2,199-214$

Leontiev, A. N.: Problems of the Development of the Mind. Moscui: Progress, 1981

LUQUE, A.: La regulación verbal en los años preescolares: una investigación con la reproducción de series. Tesis de licenciatura. Universidad de Sevilla, 1985.

Luria, A. R.: «Speech Development and the Formation of Mental Process», en M. Cole e I. Maltzman (eds.): A Handbook of Contemporary Soviet Psycbology. Nueva York: Basic Books, 1969, 121-162.

- «Desarrollo y disolución de la función directiva del habla», en A. R. Luria, R. Brain, E. H. Lenneberg y otros: Psicologia y Lenguaje. Madrid: Fundamentos, 1973.

- Lenguaje y comportamiento. Madrid: Fundamentos, 1974.

- El cerebro en acción. Barcelona: Fontanella, 1979.

- Neuropsicología de la memoria. Madrid: H. Blume, 1980.

- Conciencia y lenguaje. Madrid: Aprendizaje-Visor, 1984.

MaCCOBY, E. E.: «Developmental Psychology». Annual Review of Pscybology, 1964, 15, 202-250.

MCCABE, A. E.: «A Paradox of Self-Regulation in Speech-Motor Interaction: Semantic Degradation in Impulsive Segmentation», en G. Zivin (ed.): ob. cit.; 1979.

MEACHAN, J. A.: «Verbal Guidance through Remembergin the Goals of Actions». Child Development, 1978, 49, 188-194.

- «The Role of the Verbal Activity in Remembering the Goals of Actions», en G. Zivin (ed.): The Development of the Self-Regulation tbrough Private Speech. Nueva York: Willey, 1979, 237-263.

Meichembaum, D., y Goodman, S.: «Clinical Use of Private Speech and Critical Questions about its Study in Natural Settings», en G. Zivin (ed.): The Development of Self-Regulation through Private Speech. Nueva York: Wiley, 1979.

Miller, G. A.; Galanter, E., y Pribram, K. H.: Planes y estructuras de la conducta. Madrid: Debate, 1983.

Miller, S.; SHelton, J., y FlAVEll, J. H.: «A Test of Luria's Hypotheses Concerning the Development of Verbal Self-Regulation». Child Development, 1970, 41, 651-665.

Mischel, W., y EBBESEN, E.: «Attention in Delay of Gratification». Journal of Personality and Social Psychology, $1970,16,329-337$.

Mischel, W., y MOORE, B.: «Effects of Attention to Symbolically Presented Rewards Upon Self-Control». Journal of Personality and Social Pscybology, 1973, 28, 172-179. do. Universidad de Sevilla, 1982. 
Pascunl-Leone, J.: «La teoría de los operadores constructivos», en J. Delval (comp.): Lecturas en psicologia del niño, vol. l. Madrid: Alianza, 1978.

PATterson, C. J.: «Self-Control and Self-Regulation in Childhood», en T. M. Fields, A. Huston, H. C. Quay, L. Troll y G. E. Finley (eds.): Review in Human Development. Nueva York: Wiley, 1982.

PIAGET, J.: La formación del símbolo en el niño. México: Fondo de Cultura Económica, 1973.

- El lenguaje y el pensamiento en el niño, vol. I. Buenos Aires: Guadalupe, 1976.

REESE, H. W.: "Verbal Mediation as a Function of Age Level». Psychological Bulletin, 1962, 59, 502-509.

Saltz, E.; Campbell, S., y SxotKo, D.: «Verbal Control of Behavior: The Effects of Shouting». Developmental Psychology, 1962, 3, 461-464.

TiNSLEY, V. S., y WaTERS, H. S.: «The Development of Verbal Control over Motor Behavior: A Replication and Extension of Luria's Findings». Child Development, 1982, 53, 746-753.

VyGotsky, L. S.: Pensamiento y lenguaje. Buenos Aires: La Pléyade, 1973.

WaLLON, H.: Los origenes del pensamiento en el niño, vol. I. Buenos Aires: Lautaro, 1980.

WERTSCH, J. V.: «The Regulation of Human Action and the Given-New Organization of Private Speech», en G. Zivin (ed.): The Development of Self-Regulation through Private Speech. Nueva York: Wiley, 1979, 79-89.

WILDER, L.: «The Role of Speech and others Extra-signal Feedback in the Regulation of the Child's Sensimotor Behavior». Speech Monographs, 1969, 36, 426-434.

WozNIAK, R. H.: «Verbal Regulation of Motor Behavior-Soviet Research and No-Soviet Replications». Human Development, 1972, 15, 13-57.

Zivin, G.: «Functions of private speech during problem-solving in preschool children». Dissertation Abs. tracts International, 1972, 33 (4-B), 1834; Ann Arbor, Mich.: University Microfilms, núms. 72-26, 224.

- "Development Aspects of Rhythm in Self-Regulation», en K. E. Riegel y J. M. Meacham (eds.): The Developing Individual in a Changing Word, vol. I: «Historical and Cultural Issues». La Haya: Mouton, 1976, $161+171$. 